\title{
Diagnosis and assessment of polymyalgia rheumatica: a step forward
}

\author{
"In an effort to advance the field of polymyalgia rheumatica, an \\ international consortium undertook a multistep effort to define \\ classification criteria for this disease."
}

Polymyalgia rheumatica (PMR) is accepted as being the most common inflammatory disease in persons over the age of 50 years of European descent. The clinical features of PMR are myriad, and each of the clinical features used by clinicians in the ascertainment of the disease diagnosis are, in and of themselves, nonspecific and occur in many other disease mimickers.

No single disease feature or disease biomarker is specific for PMR, a situation that parallels that of other systemic rheumatic diseases, including rheumatoid arthritis, systemic lupus erythematosus, seronegative spondyloarthropathies and many forms of vasculitis. All of these conditions rely on a catalog of disease features for diagnosis and classification. For conditions like PMR, the diagnosis is often made on a gestalt of features, as Spiera and Westhovens offered in a recent opinion piece, where they referred to PMR as "the pornography of rheumatic diseases," that, as US Supreme Court Justice, Potter Stewart, famously stated, "I could never succeed in intelligibly doing so (defining pornography), but I know it when I see it..." [1].

In an effort to advance the field of PMR, an international consortium undertook a multistep effort to define classification criteria for this disease [2]. In developing these classification criteria, the purpose was to define key clinical characteristics that would be useful in grouping patients together with a disease syndrome identified as PMR for purposes of establishing uniformity in the patient group for further study, including enrollment of patients into clinical trials.

These classification criteria are regarded as provisional, as further validation is awaited. They are the results of a multiyear effort, which began with a conference of experts who suggested more than 50 clinical, laboratory, and imaging features potentially useful in both assessment and classification of PMR. This was followed by a systematic literature review to identify candidate criteria items and then testing of core criteria, of which seven were identified by more than $70 \%$ of the expert respondents [3]. The consensus also endorsed a step-wise approach to diagnosis utilizing core inclusion and exclusion criteria and also defined a complete response as greater than $70 \%$ patient global response to therapy $[3,4]$. The criteria elements, which are part of the classification, and the approach to disease management have been endorsed by a wider survey of family practitioners, rheumatologists and nonrheumatologists [3].

Based on these candidate criteria, a longitudinal prospective study was performed, wherein 125 patients with PMR diagnosed by rheumatology experts and community physicians and 169 non-PMR comparison subjects with conditions mimicking PMR were evaluated and followed for 6 months [2]. Entry criteria were new-onset bilateral shoulder pain and an age of over 50 years. All patients with PMR were included prior to initiation of glucocorticosteroid therapy. Mimicking conditions enrolled for the study were both inflammatory (such as newly diagnosed late-onset rheumatoid arthritis, spondyloarthritis, connective tissue diseases) and noninflammatory (such as regional shoulder conditions, osteoarthritis and fibromyalgia).

At the end of the study, resultant criteria that were found to be most useful in diagnosing PMR and discriminating PMR from other conditions were identified, and then assessed on a point system. According to this point system, a patient who entered the study with new-onset bilateral shoulder pain and was aged over 50 years who had a score of 4 points or more could be classified as having PMR so long as other mimicking conditions were excluded. Components of the scoring algorithm are morning stiffness $>45$ min (2 points), hip pain/limited range of motion (1 point), normal rheumatoid factor and/or anticitrullinated protein antibody (2 points), and absence of peripheral joint pain (1 point). A score $\geq 4$ points had $68 \%$ sensitivity and $78 \%$ specificity for discriminating all comparison subjects from PMR. The specificity
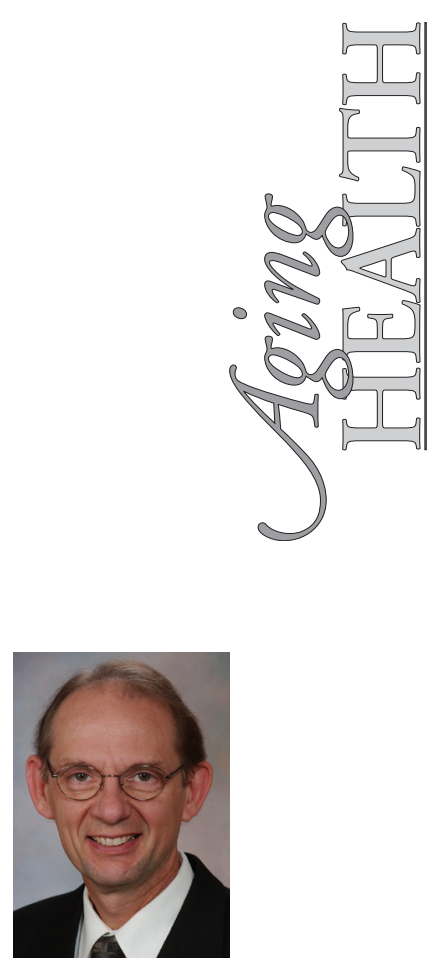

Eric L Matteson

Author for correspondence: Mayo Clinic College of Medicine, Rochester, MN 55905, USA matteson.eric@mayo.edu

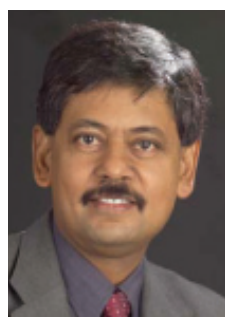

Bhaskar Dasgupta

Southend University Hospital and Essex University, Essex, UK

\section{Keywords}

- classification • criteria

- polymyalgia rheumatica

- ultrasonography

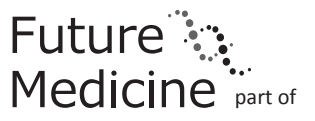


was higher $(88 \%)$ for discriminating shoulder conditions from PMR and lower (65\%) for discriminating rheumatoid arthritis from PMR. Adding ultrasound, a score of $\geq 5$ had increased sensitivity to $66 \%$ and specificity to $81 \%$.

A unique aspect of this study was the incorporation of ultrasonography to identify and evaluate features typical of PMR. Ultrasonography was found useful for classification of PMR and added to the specificity of the classification. In a follow-up manuscript, ultrasound was reported to be useful in the assessment of disease activity over the 6-month course of the study [5]. The specificity was good when comparing PMR to noninflammatory shoulder pain but was less so against rheumatoid arthritis, suggesting a genuine overlap of PMR with other forms of late-onset inflammatory arthritis.

These provisional classification criteria represent a step forward in the progress toward improvement of the recognition and management of this disease syndrome. Importantly, and unlike other criteria that are purported to be diagnostic of PMR, these criteria were systematically assessed in a large group of patients in a prospective longitudinal observational study comparing PMR with patients with mimicking diseases who had new-onset bilateral shoulder pain and were aged over 50 years.

Importantly, this set of classification criteria does not incorporate the concept of response to glucocorticosteroids as a classification feature. The use of glucocorticosteroid response as a diagnostic criterion is nonspecific, as patients who have other inflammatory conditions, including rheumatoid arthritis, may have a similar response of symptoms; and not all patients with polymyalgia may respond to conventional doses of glucocorticosteroids. Perhaps more importantly, the inclusion of glucocorticosteroid response in any diagnostic or classification criteria thwarts efforts to examine therapeutics in this disease, as an initial glucocorticosteroid response with 'good' improvement of symptoms frustrates the evaluation of other therapeutics, that have been found effective in other rheumatic diseases. Even over a 6-month period, however, response to glucocorticosteroids in the classification study did not add additional information to other clinical characteristics identified as useful in the classification of patients with PMR [2].

The new set of classification criteria may be viewed in comparison to older sets of diagnostic criteria for PMR [6-9]. All of these older criteria include the presence of proximal muscle pain and stiffness; this could be shoulder and pelvic girdle pain or shoulder or pelvic girdle stiffness, which is in contradistinction to the European League Against Rheumatism/American College of Rheumatology classification criteria, which call for bilateral shoulder pain as an essential feature. As such, it is possible that a small percentage of patients who may otherwise be diagnosed with PMR who have hip stiffness but do not have shoulder involvement would not be included in a study of patients, which was based on the new provisional classification criteria. However, isolated hip pain without shoulder pain is often due to common spinal, circulatory, arthritic conditions and these new classification criteria secure a more homogeneous patient population that is more appropriate for clinical study.

None of the previous classification criteria for PMR incorporate imaging. Since these older criteria were published, further studies evaluating MRI and ultrasonography have identified features which are typical, but not diagnostic, of PMR [10]. Most useful among these for clinical application is ultrasonography demonstrating bilateral subacromial/subdeltoid bursitis in virtually all patients with PMR [2,5].

An important aspect of the classification study, which is useful clinically, is the confirmation of the diagnosis on follow-up. In this regard, the condition most likely to be confused with PMR is early onset rheumatoid arthritis. The follow-up of these patients over this 6-month period was important, with the observation that the clinical features best discriminating rheumatoid arthritis from PMR were peripheral synovitis, the presence of rheumatoid factor and/or anticitrillunated peptide antibody and hip pain/limited hip range of motion [3]. The features that best discriminated any other shoulder condition, such as new-onset bilateral rotator cuff syndromes, generalized osteoarthritis, and other conditions including a variety of endocrinopathies, infection and fibromyalgia, were hip pain/limited hip range of motion, morning stiffness, and elevated C-reactive protein and/or erythrocyte sedimentation rate [3].

The new classification criteria for PMR are best used in the evaluation of patients over the age of 50 years with new-onset (less than 12 weeks) bilateral shoulder pain and abnormal acute-phase reactants. As with the criteria for other conditions, such as rheumatoid arthritis and systemic lupus erythematosus, the same caveat holds that the criteria apply in the absence of competing diagnoses.

PMR remains a frustratingly difficult condition to diagnose and classify because of the heterogeneity at presentation, the variable disease 
course, and overlap with other conditions. The sensitivity $(66 \%)$ and specificity $(81 \%)$ of the new classification criteria for discriminating all comparison subjects from PMR, while being a step forward, also highlight knowledge gaps in this disease, and the future research agenda. These uncertainties highlight the need for an early confirmation of a diagnosis of PMR in a specialist rheumatology setting before long-term steroid therapy is planned.

Future goals in the study of PMR include development of more robust response criteria, studies of comorbidities including cardiovascular disease, metabolic disease, malignancy, mortality and the genomic characterization of PMR. With the development of new therapeutics, pharmacogenomic characterization of the disease and response variables, including development

\section{References}

1. Spiera R, Westhovens R. Provisional diagnostic criteria for polymyalgia rheumatica: moving beyond clinical intuition? Arthritis Rheum. 64(4), 955-957 (2012).

2. Dasgupta B, Cimmino MA, Maradit-Kremers $\mathrm{H}$ et al. 2012 provisional classification criteria for polymyalgia rheumatica: a European League Against Rheumatism/American College of Rheumatology collaborative initiative. Arthritis Rheum. 65(4), 943-954 (2012).

3. Dasgupta B, Salvarani C, Schirmer M et al.; members of the American College of Rheumatology Work Group for Development

of better biomarkers for disease activity and severity, will become increasingly important. Ultimately, new and improved therapeutics will be developed and tested, which should serve to shorten the disease course and reduce the burden of long-term glucocorticosteroid therapy.

\section{Financial \& competing interests disclosure}

The classification study for polymyalgia rheumatica was sponsored by the American College of Rheumatology and the European League against Rheumatism with support from Mayo Clinic. The authors have no other relevant affiliations or financial involvement with any organization or entity with a financial interest in or financial conflict with the subject matter or materials discussed in the manuscript apart from those disclosed.

No writing assistance was utilized in the production of this manuscript.

of Classification Criteria for PMR. Developing classification criteria for polymyalgia rheumatica: comparison of views from an expert panel and wider survey. J. Rheumatol. 35, 270-277 (2008).

4. Dasgupta B, Matteson EL, Maradit-Kremers $\mathrm{H}$. Management guidelines and outcome measures in polymyalgia rheumatica (PMR). Clin. Exp. Rheumatol. 25(Suppl. 47), S130-S136 (2007).

5. Matteson EL, Maradit-Kremers H, Cimmino MA et al. Patient-reported outcomes in polymyalgia rheumatica. J. Rheumatol. 39(4), 795-803 (2012)

6. Chuang TY, Hunder GG, Ilstrup DMARDs, Kirkland LT. Polymyalgia rheumatica: a 10 year epidemiologic and clinical study. Ann. Intern. Med. 97, 672-680 (1982).

7. Healey LA. Long-term follow-up of polymyalgia rheumatica: evidence for synovitis. Semin. Arthritis Rheum. 13, 322-328 (1984).

8. Bird HA, Esselinckx W, Dixon ASJ, Mowat AG, Wood PHN. An evaluation of criteria for polymyalgia rheumatica. Ann. Rheum. Dis. 38, 434-439 (1979).

9. Jones JG, Hazleman BL. Prognosis and management of polymyalgia rheumatica. Ann. Rheum. Dis. 40, 1-5 (1981).

10. Breuer GS, Nesher G. What does imaging tell us about polymyalgia rheumatica? Rheumatology 51, 5-6 (2011). 